\title{
NIVELES DE CONOCIMIENTOS LINGÜÍSTICOS EN LA SEGUNDA LENGUA (L2) EN ESTUDIANTES DE MAGISTERIO EIB EN BILWI
}

\author{
Cándida Rugama Pérez ${ }^{[6]}$ \\ Débora Bravo Alfaro ${ }^{[7]}$ \\ Argentina García Solórzano ${ }^{[8]}$
}

\section{Resumen}

Esta investigación sobre los niveles de conocimientos lingüísticos es de carácter cualitativo. El propósito es contribuir al mejoramiento de las estrategias metodológicas utilizadas para atender los niveles de conocimientos lingüísticos en el español como segunda lengua que posee la comunidad estudiantil del primer año de magisterio de Educación Intercultural Bilingüe -EIB-, en la escuela Normal "Gran Ducado de Luxemburgo" segundo semestre del año escolar 2006.

Para este propósito se analizaron algunos elementos metodológicos que contribuyen al desarrollo de los diferentes niveles de conocimientos lingüísticos en el español como segunda lengua; luego, posteriormente se comparten algunas recomendaciones metodológicas que ayudarían al desarrollo de competencias de acuerdo a los niveles de conocimientos lingüísticos que poseen las y los estudiantes.

En esta investigación se identifica un nivel al que se le denomina semi-principiante, y está por debajo de los requisitos del último nivel de conocimientos lingüísticos que plantean algunos lingüistas.

Se recomienda adecuar los programas del español como segunda lengua para que responda a los intereses y necesidades de estudiantes de habla miskitu, utilizando diferentes estrategias y técnicas didácticas que faciliten una atención diferenciada según los niveles de conocimientos lingüísticos.

[6] Licda. en Educación Intercultural Bilingüe, URACCAN, Bilwi.

[7] Licda. En Educación Intercultural Bilingüe,URACCAN, Bilwi..

[8] Máster en Estudios Interdisciplinarios, URACCAN, Bilwi.Tutora de la investigación

48 | CIENCIA E INTERCULTURALIdAd, Volumen 4, Año 2, No. 2, Junio 2009 


\section{Introducción}

La educación en la Escuela Normal "Gran Ducado de Luxemburgo", se imparte en idioma español de conformidad con el currículo nacional. Se ha obviado que esta región es multilingüe y multicultural, que hay muchos estudiantes cuya lengua materna no es el español, sino que se han visto como simples receptores, ya que su problema lingüístico con el español nos les ha permitido un mejor desempeño en las aulas de clase.

Se ha escrito sobre la enseñanza de la segunda lengua, específicamente con relación a factores de orden metodológico que limitan el aprendizaje de esa asignatura, lo que procura mayor importancia a los resultados de este estudio, ya que servirá como un documento base para continuar profundizando sobre la atención brindada en los niveles lingüísticos de cada estudiante.

La investigación se realizó en la Escuela Normal "Gran Ducado de Luxemburgo" en el I semestre del 2006 con estudiantes de primer año de magisterio como requisito para optar al título de licenciadas en Educación Intercultural Bilingüe. Esta oportunidad permitió el descubrimiento de niveles de conocimientos lingüísticos que poseen las y los estudiantes al llegar a primer año de magisterio, esos niveles están por debajo de la clasificación hecha por diferentes autores, lo que nos lleva a reflexionar sobre la manera cómo se está tratando los niveles de conocimientos lingüísticos que llevan consigo estas personas estudiantes.

El estudio ha permitido el planteamiento de algunas estrategias metodológicas que podrían ayudar a la enseñanza - aprendizaje del español como segunda lengua para brindar una mejor atención estudiantil de acuerdo a los diferentes niveles de conocimientos lingüísticos. Eso contribuirá a mejorar las competencias lingüísticas tanto en español como en la lengua miskitu en cuanto a habilidades de escucha, expresión oral, lectura y escritura.

Como objetivos se plantearon la valoración de los niveles de conocimientos lingüísticos en el español como segunda lengua que poseen las y los estudiantes del primer año de magisterio. De acuerdo a estos conocimientos se analizaron los elementos metodológicos que contribuyen al desarrollo de los diferentes niveles de conocimientos lingüísticos y compartiremos recomendaciones metodológicas para el desarrollo de competencias en el español como segunda lengua. 


\section{EDUCACIÓN INTERCULTURAL BILINGÜE EN LAS REGIONES AUTÓNOMAS ATLÁNTICO NORTE Y ATLÁNTICO SUR}

\section{Revisión de literatura}

Algunas de las literaturas relevantes encontradas que ayudan al soporte científico del tema investigado son:

En su obra Pedagogía en Píldora (Solá; 1989) sostiene que el docente "es un modelo viviente, al que sus alumnos observan, estudian e imitan en todos sus rasgos. Los alumnos reproducen de sus profesores(as), su modo de hablar, sus modismos, los rasgos de su escritura, su modo de sentir, su modo de juzgar".

En esa misma obra, expresa además que: "cuando la integración maestro(a) alumno(a) es adecuada, propicia, además de la adquisición de conocimientos, otras actitudes en los educandos, como interés en sus estudios, fomenta el trabajo sistemático y estimula el pensamiento lógico. Y así, consigna que la metodología señala el encadenamiento lógico de ejercicios graduados para obtener un fin, éste puede ser, la adquisición de conocimientos".

Al respecto de los niveles lingüísticos, Carvajal (2000) en su investigación titulada Niveles de conocimientos lingüísticos, citó que: "existen diferentes niveles lingüísticos en el aprendizaje de una segunda lengua, que se les denomina: nivel principiante, nivel intermedio, nivel avanzado y nivel superior y se explican de la siguiente manera".

Fernández $(2,000)$, plantea que: "La lingüística es la ciencia que se ocupa de la descripción y explicación de los hechos del lenguaje en sus distintos niveles: fónico, léxico y sintáctico." Además expresa que "Trabajar las cuatros habilidades fue casi un dogma para los estructuralistas, aunque en 1880 nació una tradición que pretendía favorecer el habla desde el principio del proceso de aprendizaje."

Freeland (2000), en su investigación sobre Lenguaje indicó que: "en un contexto donde la lengua materna no es el español, éste se habla como segunda lengua o $L_{2}$; es el nuevo idioma que tiene que aprender por ser la lengua oficial, a nivel nacional o por alguna otra consecuencia, y ser utilizada ya sea en la escuela, la iglesia, el parque o en el contexto social donde se desarrolla intelectual y físicamente".

Venezia (2003) confirmó que: "el Bilingüismo es el uso directo - activo y pasivo - de dos o más lenguas por parte de un hablante directo. Es una característica del uso de las lenguas, y por lo tanto, se refiere al habla." Asimismo, precisó que: "lengua materna es la primera en la cual una persona aprende a hablar y que en la mayoría de los casos mantendrá el predominio sobre el aprendizaje de otras lenguas".

50 | CIENCIA E INTERCULTURALIDAd, Volumen 4, Año 2, No. 2, Junio 2009 
En un estudio realizado sobre Vida Escolar, Good y Weinsten (2005) plantean que "la escuela es el lugar en que los niños y las niñas adquieren o no diversas competencias que llegan a definir buena parte de su autoestima, donde se forman amistades con los compañeros y donde se practica el papel de miembro de la comunidad; todo lo anterior en un período de desarrollo muy significativo".

\section{Materiales y métodos}

El estudio es descriptivo-propositivo, con un enfoque cualitativo y de corte transversal. Es descriptivo-propositivo debido a que se va a contribuir al mejoramiento de las estrategias metodológicas utilizadas para elevar los niveles de conocimientos lingüísticos en el español como segunda lengua en estudiantes del primer año de magisterio de EIB en la Escuela Normal "Gran Ducado de Luxemburgo".

\section{Variables:}

- Diferentes niveles de conocimientos lingüísticos;

- El lenguaje español como segunda lengua;

- Estrategias metodológicas de enseñanza del español como segunda lengua.

- Se utilizaron como instrumentos: Guía de entrevista a docentes de lengua miskitu y español, guía de entrevista a directora de la Escuela Normal "Gran Ducado de Luxemburgo", guía de entrevista a estudiantes, un test de evaluación y una guía de observación.

\section{Procedimiento de recolección de datos}

Para la aplicación de cada uno de los instrumentos se realizó un diálogo que permitió entrar en un clima de confianza con los/las estudiantes y con los docentes objeto de estudio. Las entrevistas se realizaron en un margen de tiempo aproximado de 15 minutos, las observaciones se realizaron en períodos de clase de 90 minutos.

\section{Procesamiento de datos}

Después de aplicar los instrumentos, se ordenaron las respuestas por preguntas, se clasificaron por objetivo y por fuente. Su procesamiento se realizó utilizando la técnica del palote en el caso del test y entrevistas a docentes, a la directora y a estudiantes. Posteriormente se categorizaron los resultados, para proceder al análisis, 


\section{EDUCACIÓN INTERCULTURAL BILINGÜE EN LAS REGIONES AUTÓNOMAS ATLÁNTICO NORTE Y ATLÁNTICO SUR}

interpretación, discusión de resultados, conclusiones y recomendaciones de acuerdo a los objetivos propuestos.

\section{Resultados y discusión}

\section{Niveles de conocimientos lingüísticos}

Los niveles de conocimientos lingüísticos encontrados en la comunidad estudiantil fueron tres, pero se definen cuatro ya que el único nivel que no fue encontrado es el superior:

Semi-principiantes: Se denominó semi-principiantes a las personas estudiantes que no realizaron todo el test de evaluación, solamente escribieron el nombre de su papá, de su mamá, su propio nombre pero aún lo escribieron con dificultad. Sus habilidades fueron limitadas, González E. (2004:35) le llama Nivel - Usuario Elemental, comprende palabras, nombres conocidos y frases muy sencillas, por ejemplo, las que hay en letreros, carteles y catálogos.

Podría decirse que están en ese nivel de semi-principiantes por una inadecuada atención del español como segunda lengua, poca lectura, o bien, su capacidad lingüística para el español fue poco o nada estimulada en la escuela de Educación Primaria y en el hogar.

El nivel principiante: En este nivel encontramos aquellas y aquellos estudiantes que escribieron su nombre, el de su papá, el de su mamá y realizaron algunas partes del test de evaluación pero con palabras no bien redactadas. Para Carvajal M. (2000:9) "los que están en este nivel se desempeñan en actividades muy predecibles de la vida diaria, pueden producir solamente oraciones ya formuladas, listados y enumeraciones. Pueden leer algunas oraciones o frases, es muy difícil de entenderlos, incluso para aquellas personas que están acostumbradas a tratar con extranjeros".

Estos estudiantes pueden ejercitar la lectura silenciosa y oral; la transcripción de pequeños textos, para mejorar las habilidades que ya tienen desarrolladas y poder lograr el alcance del nivel intermedio. Estos estudiantes desarrollaron más su capacidad lingüística lo que les ha ayudado a entender, interpretar y responder un poco más que los del nivel anterior. Como los del nivel anterior, también requieren una atención especial para que gradualmente vayan alcanzando un mayor nivel de conocimientos lingüísticos y pueden ser competitivos ante cualquier situación que se les presente.

52 | CIenCIA E INTERCULturalidad, Volumen 4, Año 2, No. 2, Junio 2009 
El nivel intermedio: En este nivel ubicamos aquellas y aquellos estudiantes que realizaron los ejercicios propuestos de manera mecánica, ya que a veces se valían de otras compañeras y compañeros para completar cada una de las actividades que se orientaban en la evaluación, además presentaban bastantes problemas de caligrafía y ortografía.

Según Carvajal M., (2000:9), "los de este nivel pueden mantener una conversación simple cara a cara preguntando y respondiendo preguntas sencillas. Se desempeñan en algunas situaciones informales con bastantes limitaciones". Con estos grupos de estudiantes se pueden poner en práctica las reglas de ortografía y caligrafía ya que ese es su problema más sentido. Requieren de esfuerzo, bastante trabajo y atención para seguir teniendo mejor nivel de conocimientos lingüísticos.

El nivel avanzado: Están quienes lograron completar todo el test de evaluación con pocos problemas de ortografía, pero sí con algún problema lingüístico. Según Carvajal M. (2000:9) “estos se desempeñan en la mayoría de situaciones informales y en algunas situaciones formales. Pueden describir y narrar en marcos más amplio de tiempo y aspecto. Personas que no están acostumbradas a tratar con no nativos, pueden entenderlos".

En esta categoría de estudiantes se pueden desarrollar habilidades y destrezas en el español como segunda lengua para ir escalando hacia el nivel superior y lograr las competencias lingüísticas en ambas lenguas, la materna y la lengua oficial del país.

El nivel superior: No fue detectado en el grupo. Según Carvajal M. (2000:9): "pueden desempeñarse tanto en situaciones formales como no formales. Pueden discutir ampliamente, justificar sus opiniones y formular situaciones hipotéticas. Que casi no tienen interferencia en la comunicación y no les incomodan los hablantes nativos. Participan en discursos extensos". Pudimos descubrir que las/los estudiantes presentan diferentes niveles de conocimientos lingüísticos y hacen gran esfuerzo por cumplir con todas las tareas que se les orienta.

Aunque docentes que imparten las clases de lenguas en su mayoría se consideran muy buenos facilitadores, abnegados a la profesión; sin embargo, obvian los diferentes niveles lingüísticos que posee el grupo de estudiantes y aplican las mismas estrategias y técnicas pedagógicas sin atención diferenciada.

Aunque se atiende a la comunidad estudiantil para apoyarles en su aprendizaje, se ve la necesidad de brindarles una atención diferenciada de acuerdo a los niveles que cada estudiante presenta. Además, presentan problemas de caligrafía y ortografía 


\section{EDUCACIÓN INTERCULTURAL BILINGÜE EN LAS REGIONES AUTÓNOMAS ATLÁNTICO NORTE Y ATLÁNTICO SUR}

y esto es desapercibido por algunos docentes, según Solá M (1989:26), quienes se olvidan que los estudiantes reproducen su modo de hablar, sus modismos, los rasgos de su escritura, su modo de sentir y hasta su modo de juzgar.

En su mayoría, los estudiantes manifestaron que la clase que más les gusta es el miskitu, porque entienden lo que hacen, saben lo que les orientan los docentes, saben lo que están hablando, pero quieren aprender el español, aunque lo sienten algo difícil.

De acuerdo con los datos recaudados, no se encuentra ningún estudiante en el nivel superior; pero, se logró descubrir que no solamente existen los cuatro niveles de conocimientos lingüísticos que manifiesta Carvajal M. (2000:9), sino que también el semi-principiante de acuerdo a los datos obtenidos de las observaciones, entrevistas a docentes y test de evaluación aplicados a las y los estudiantes objeto de estudio.

\section{Ambiente Familiar}

La mayoría de personas estudiantes entrevistadas manifestaron que estudiaron en escuelas de Educación Primaria Bilingües y que pertenecen a la lengua materna miskitu y por ende su lengua materna es miskitu, ya que su papá, su mamá, abuelito, abuelita hablan la misma lengua.

Según Solá M. (1989:44), los problemas emocionales que afectan la personalidad del educando pueden perjudicar tanto el rendimiento como el comportamiento escolar. Sin embargo, esos problemas pueden afectar de tal modo la vida del estudiante, que llegan a dar la impresión de que se trata de un caso de poca inteligencia, cuando en realidad se trata de un estudiante normal.

\section{Ambiente Escolar}

El ambiente en que reciben las clases no es motivador, hace falta energía eléctrica para desarrollar las clases específicamente en los tiempos de lluvias, la pizarra está en malas condiciones, se escribe con tizas y además no todos los estudiantes cuentan con pupitres, falta decoración y materiales de trabajo como grabadoras, cassets, entre otros. Good y Weinsten (1986: 34) plantean que la escuela es el lugar en que la niñez adquiere o no diversas competencias que llegan a definir buena parte de su autoestima, donde se forman amistades, donde se practica el papel de miembro de la comunidad; todo lo anterior en un periodo de desarrollo muy significativo.

Además expresa que el hogar y la escuela son los espacios en donde el nuevo individuo se forma a través de la interacción con el objeto de estudio en interacción 
con los demás. Toda la niñez se debe convertir en sujetos de aprendizaje, capaces de construir sus propios conocimientos gracias a la acción oportuna de los docentes facilitadores en la escuela.

En un contexto donde se practica la interculturalidad y el bilingüismo, los docentes deben de poner en práctica un modelaje acorde a la necesidad de aprendizaje de las y los estudiantes, como es el aprendizaje de una segunda lengua. La educación sería la acción recíproca de dos sujetos, uno con respecto al otro, que conduciría a la transformación de dos personalidades presentes. Dentro del proceso de la educación la transmisión de conocimientos se debe dar de forma horizontal, de manera gradual y sistemática para poder establecer la acción recíproca entre dos sujetos.

El aula de clase debe de reflejar un ambiente de interculturalidad de educación bilingüe donde deben de existir rincones de aprendizaje, murales, carteles, afiches y pensamientos que faciliten al estudiante vivir en constante relación para la familiarización con palabras nuevas de la segunda lengua o lengua oficial del país, como es el español.

\section{Aspecto Pedagógico - Metodológico}

La docente manifestó que los programas están elaborados de acuerdo a la realidad del contexto sociocultural de los estudiantes y además se caracterizan por ser flexibles y poderlos adecuar a las necesidades educativas e intereses de los estudiantes.

Las visitas pedagógicas no las realiza nadie, a veces a lo interno se promueven intercapacitaciones y la Sede Central -MINED- acorde a especialidad. Los docentes no han sido capacitados hasta el momento. Las dificultades que presentan es que no tienen materiales ni suficientes ni adecuados, que garanticen la efectividad en el aprendizaje.

En el aula, raras veces se atienden las diferencias individuales. Para esto se requiere de tiempo extra, mucha conciencia y voluntad, tanto del docente como del estudiante. El aprendizaje cooperativo es una de las estrategias de enseñanza, esta técnica requiere que los estudiantes trabajen juntos en grupos de habilidades diversas. El aprendizaje cooperativo hace sentirse miembro activo durante el proceso enseñanza - aprendizaje, esto supera en gran parte esas diferencias individuales.

Los docentes de lenguaje no se capacitan pero han cursado estudios universitarios, donde han estudiado la lengua que desarrollan. Aunque Según Solá M. (1989:44) "El maestro(a) debe tener sano criterio, mente despejada, penetración psicológica, 


\section{EDUCACIÓN INTERCULTURAL BILINGÜE EN LAS REGIONES AUTÓNOMAS ATLÁNTICO NORTE Y ATLÁNTICO SUR}

buena cultura y estudiar constantemente los adelantos de la ciencia y las rutas de la pedagogía”.

La metodología que se aplica para la enseñanza del español como segunda lengua dicen los docentes que se basa en la metodología activa participativa. En las visitas realizadas a pie de aula, se observó que los docentes de lengua procuran mantener un ambiente óptimo con un clima de familiaridad, para que el estudiante se sienta en confianza, pero se considera que se necesita además de muchas técnicas y estrategias, material didáctico relacionado al tema que se impartirá para que haya motivación y se logre un aprendizaje que los lleve a dominar el nivel superior lingüístico.

Según lo dicho por Carvajal M. (2000:9), los de nivel superior: "Pueden desempeñarse tanto en situaciones formales como no formales. Pueden discutir ampliamente, justificar sus opiniones, formular situaciones hipotéticas. Los errores casi no interfieren en la comunicación y no les incomodan a los hablantes nativos. Participan en discursos extensos".

En las aulas de clase se observó la ausencia de rincones de aprendizajes y otros recursos como murales, láminas, tarjetas, etc., que son necesarios para lograr la retención memorística de aprendizajes relacionados al español como segunda lengua, ya que se necesita que se pongan en práctica porque esto ayudaría a la comunidad estudiantil a retener más y ser más participativos en el proceso de enseñanza - aprendizaje que se les propician.

Se considera necesario que los programas del español como segunda lengua deben ser ajustados a las necesidades, intereses y niveles de conocimientos lingüísticos que posee cada estudiante. Según Venezia P.(2003:25) afirma: "El Bilingüismo es el uso directo - activo y pasivo - de dos o más lenguas por parte de un hablante directo".

\section{Preferencias por asignaturas}

Las asignaturas de mayor preferencia para las y los estudiantes entrevistados es el mískitu, porque es su propio idioma y el español porque lo quieren aprender. Esto nos confirma que tienen el deseo de aprender una segunda lengua, y cuando esto sucede el aprendizaje se vuelve significativo, lo que lo lleva a lograr su propósito.

Para que la asignatura se vuelva interesante es necesario valerse de diferentes formas de presentar la clase: variando las actividades, haciéndolas atractivas con el uso de diferentes materiales didácticos.

56 | CIenCia E InTERCULturalidad, Volumen 4, Año 2, No. 2, Junio 2009 
A veces los reglamentos y las normas establecidas van muy divorciadas de la situación que se vive en un centro educativo, hacemos cumplir lo predicado por el documento y se pasan desapercibidas las necesidades de aprendizajes e intereses de la comunidad estudiantil, lo único que provoca esa rigidez es el descontento, bajo rendimiento académico, indiferencias por algunas disciplinas de estudio y la baja autoestima en las aulas de clases.

\section{Conclusiones}

Los niveles de conocimientos lingüísticos de aquellas y aquellos estudiantes del primer año de magisterio en la escuela Normal son bajos en términos generales, lo que afecta su desempeño estudiantil y por una atención inadecuada podrían caer en la frustración o bien, en el rechazo al aprendizaje.

El ingreso de los estudiantes procedentes de escuelas bilingües de las comunidades indígenas al primer año de magisterio es traumático y frustrante por el bajo nivel lingüístico alcanzado en la segunda lengua $-\mathrm{L}_{2}$

El nivel lingüístico detectado en algunos casos es tan bajo que ni siquiera se contempla en la clasificación hecha por los teóricos, nivel que definimos como semiprincipiante.

Los elementos metodológicos encontrados en el desarrollo de las clases, realmente no contribuyen al desarrollo de los diferentes conocimientos lingüísticos en el español como segunda lengua, a pesar de que los docentes cuentan con programas pertinentes.

Solamente con la aplicación de estrategias y técnicas didácticas adecuadas para la L2 puede contribuir al desarrollo de las competencias lingüísticas.

\section{Propuesta psicopedagógica}

\section{Metodologías participativas para la enseñanza-aprendizaje de una segunda lengua}

El método endogenésico: es uno de los apropiados para lograr un aprendizaje activo, se caracteriza porque parte de algo propio de la persona, que lo identifica y está familiarizado con el individuo, como su propio nombre y con otros elementos que forman parte de su vida diaria, como son actividades del contexto sociocultural del estudiante. 


\section{EDUCACIÓN INTERCULTURAL BILINGÜE EN LAS REGIONES AUTÓNOMAS ATLÁNTICO NORTE Y ATLÁNTICO SUR}

Según Zamora R. San José Costa Rica: El Método endogenésico (1989: Pág. 9) Nos dice que el método endogenésico procede del interior (parte de adentro, de las experiencias vividas, de lo propio). Es un método natural porque parte de las estructuras idiomáticas que posee la niñez al ingresar a la escuela.

Así como las niñas y los niños aprenden a hablar más o menos rápidamente, también escribirán y leerán con mayor o menor rapidez, pero lo harán con plena comprensión y se expresaran con independencia, seguridad, originalidad, compresión y belleza.

Aplicar el método endogenésico para la enseñanza del español como segunda lengua es efectivo. Este método ha sido utilizado por docentes que han egresado de la escuela Normal en comunidades donde existen personas que no han aprendido a leer y escribir por su problema lingüístico y se logra que aprendan a leer y a escribir, y a la misma vez logren el dominio del español como segunda lengua.

Las actividades que se realizan ayudan a motivar al estudiante, ya que escribir su nombre para toda y todo principiante es un éxito.

Cuando el estudiante lee y escribe bien su nombre, se añade al nombre el primer apellido, también escribirlo y leerlo. Luego agregar el segundo apellido. Ejemplo: Luis Kiapa. Después se le agrega el segundo apellido, Luis Kiapa Valenzuela.

Se introduce la expresión: "me llamo", estos ya son nuevos elementos que van conociendo en la escritura, pero que cotidianamente los usamos, cuando nos expresamos verbalmente.

Así sucesivamente se le van agregando otros elementos como en el ejemplo que se presenta a continuación:

"Me llamo Florita Saiman Winston". Siempre tienen que ver, leer y escribir la frase completa.

Después se introduce el "yo" y se hace el procedimiento anterior:

Ejemplo: Yo me llamo Adolfina Brown Celso.

El ejercicio se continúa también con el nombre propio de los demás estudiantes y pedir que lo pronuncien claramente y seguir el mismo procedimiento.

58 | CIENCIA E INTERCULTURALIDAd, Volumen 4, Año 2, No. 2, Junio 2009 
Se hace un listado de todos los nombres de las personas estudiantes y deberán pegarlos en la pared para que los lean sistemáticamente.

Así sucesivamente se van incluyendo los nombres de los papás, mamás, hermanas, hermanos y del docente de la escuela y escribir el se llama o ella se llama. También se escribe y pronuncia el nombre de los animales que tiene en su hogar, sus juguetes. El nombre de las instituciones, de tiendas, de todos los lugares más conocidos y populares.

Con todas esas actividades, se conoce un sin número de vocabulario y ese es el objetivo: partir de lo cotidiano para que el aprendizaje permanezca en todo momento.

Se pueden hacer murales donde se dibujen objetos con los nombres en miskitu y español. Centros de aprendizajes con canciones, pensamientos, adivinanzas, refranes. Comparaciones entre un objeto y el nombre del mismo objeto. Esta clase de actividades ayudan a "la memorización significativa y no a una memorización que se logra en el momento y después se olvida". Estas actividades se recomiendan, ya que son las que más les gusta a la comunidad estudiantil porque la clase se convierte en un ambiente ameno y óptimo que invita al aprendizaje. Con la frase: "Nadie aprende en un ambiente amenazante".

Rincones 0 centros de aprendizaje: Permanecen expuestos, donde las y los estudiantes tenga la oportunidad de familiarizarse con los contenidos en estudio. Desarrollan potenciales lingüísticos, psicomotores, socio afectivos y cognoscitivos.

Canciones: Las canciones son composiciones en versos, que se pueden cantar o hacerlas a propósito para que se le pueda impartir una clase dinámica y motivadora. Este elemento es uno de los más recomendados como técnica pedagógica para la enseñanza de una segunda lengua, ya que por medio de ellas se logra el aprendizaje de un sin número de vocabulario.

Esta canción es muy popular y se viene cantando de generación en generación, además se pueden cantar otras canciones conocidas por la comunidad estudiantil.

Ejemplo: "Tengo una muñeca"

Tengo una muñeca

Vestida de azul

Con su camisita y velo de tul

La saqué a paseo

Y se me resfrió

La tengo en la cama 


\title{
EDUCACIÓN INTERCULTURAL BILINGÜE EN LAS REGIONES AUTÓNOMAS ATLÁNTICO NORTE Y ATLÁNTICO SUR
}

\author{
Con mucho dolor. \\ Esta mañanita la lleve al doctor \\ me le dio jarabe con un tenedor. \\ Dos y dos son cuatro, cuatro y \\ dos son seis, seis y dos son \\ ocho, y ocho dieciséis. \\ Brinca la tablita, bríncala otra vez \\ Brinca la tablita, yo ya la brinqué \\ Ya verás muñeca si te curo yo.
}

Pensamientos: Conjunto de ideas propias de una persona o de una colectividad. Los pensamientos se pueden repetir en forma de juego, para que se logre su memorización para enriquecer el vocabulario. Se pueden retomar las ideas de los personajes célebres y se pueden dramatizar o se representan por medio de dibujos para su mejor interpretación.

Ejemplo: "Si pequeña es la patria, uno grande la sueña".

\section{Rubén Darío (Autor nicaragüense)}

Esta expresión la podemos analizar con la comunidad estudiantil y representa un valor cívico.

Adivinanzas: Son juegos de palabras que tienen como propósito descifrar un enigma mediante la intuición del acertijo. Esta técnica les ayuda porque en todo momento ponen en práctica esta dinámica, en la escuela, en los hogares, cuando visitan a sus amistades y en todo momento.

Se pueden hacer uso de adivinanzas que conozcan mejor. Aquí se puede dibujar el sol y escribir su nombre, además ellos pueden darle un nombre propio al sol y escribirlo con la ayuda de la persona facilitadora.

Ejemplo: Un señor muy encumbrado

que anda mejor que el reloj

se levanta muy temprano

y se acuesta a la oración.

60 | CIenCia e interCulturalidad, Volumen 4, Año 2, No. 2, Junio 2009 
Refranes: Dicho agudo, sentencioso y popular que expresa una enseñanza de validez general. Estas pueden ponerse en práctica en cualquier momento de las clases como forma de motivación y a la misma vez que repitan las palabras que contienen para ejercitar el vocabulario.

Analizar el mensaje que orienta el refrán o la moraleja, que sirve para la formación de la personalidad del individuo.

Ejemplo: Camarón que se duerme, se lo lleva la corriente.

Nuestro propósito es que se conozcan varias técnicas que propicien de manera dinámica el aprendizaje de una segunda lengua sin frustrar el proceso enseñanza - aprendizaje y que tanto para el docente y el estudiante se sientan en dinamismo y lograr un objetivo con el fin de alcanzar una meta como es el aprendizaje de una segunda lengua.

\section{Lista de referencias}

Ajá F, J. (2000). Didáctica de las lenguas o segunda lenguas. Océano, Grupo Editorial. Barcelona, España.

Betancourt, A.M. (2002). Pedagogía de la Ternura. Formación Inicial de docentes C. A. Educación Primaria o Básica.

Carvajal V, M. (2000). Niveles de conocimientos lingüísticos. Costa Rica.

Cramer, A. (1992). Lengua y culturas vivas. Educación Intercultural Bilingüe en el Ecuador.

Carreño H, F. (S/f). Enfoques y principios teóricos de la Evaluación. Trilla, México.

Enciclopedia, (1998). Manual de la Educación. Editorial Océano.

Freeland, J. (2000). Lengua. Managua, Nicaragua IPILC. URACCAN.

González B G, E. (2004). La interlengua. Interlingüísta y niveles de conocimientos lingüísticos. No. 15, 675, 684, Madrid, Edelsa.

Good y Weinsten (2005). Vida Escolar. 


\section{EDUCACIÓN INTERCULTURAL BILINGÜE EN LAS REGIONES AUTÓNOMAS ATLÁNTICO NORTE Y ATLÁNTICO SUR}

López A. (1997). Sociología de la educación. San José C.R., EUNED.M,

Solá M. J. (1989). Pedagogía en Píldora. Editorial Trillas, México.

Torres M. H. (2002), Didáctica General. Cartago, Costa Rica. Impresora Obando.

Venezia, P. (2003). Educación Intercultural y Plurilingüe. Terranova.

Zamora R. (1989). El Método endogenésico. San José Costa Rica.

62 | CIenCia E InTERCULturalidad, Volumen 4, Año 2, No. 2, Junio 2009 